\title{
Isotropic diffraction-limited focusing using a single objective lens
}

\author{
Emeric Mudry, Eric Le Moal, Patrick Ferrand, Patrick C. Chaumet, and Anne Sentenad \\ Institut Fresnel, CNRS, Aix-Marseille Université, \\ Ecole Centrale Marseille, Campus de St Jérôme, 13013 Marseille, France
}

\begin{abstract}
Focusing a light beam through a lens produces an anisotropic spot elongated along the optical axis, because the light comes from only one side of the focal point. Using the time-reversal concept, we show that isotropic focusing can be realized by placing a mirror after the focal point and shaping the incident beam. This idea is applied to confocal microscopy and brings about a dramatic improvement of the axial resolution.
\end{abstract}

PACS numbers: 41.20.-q Applied classical electromagnetism, 42.15.Dp Wave fronts and ray tracing, 07.60.Pb Conventional optical microscopes

With the ever growing importance of high resolution imaging, lithography, data storage or particle manipulation, the problem of focusing light beams into subwavelength volumes has become a major challenge. Numerous studies have been devoted to the development of novel lenses [1, 2] in conjunction (or not) with beam shaping 3 6] that permit one to reduce the size of the spot, possibly beyond the diffraction limit. Yet, these works were essentially interested in the reduction of the spot transverse dimensions and overlooked the axial dimension along the normal to the lens (the optical axis). Except for the evanescent spots that are bound to a surface [3], the axial extension is at least 3 times larger than the transverse ones [7, p. 491]. Since the three-dimensional resolution of any optical device using focused beams is dictated by the largest dimension of the spot, it appears to be of crucial importance to be able to focus light into spherical volumes.

The fundamental reason why the intensity distribution of the light focused through a lens has the shape of a cigar is that the illumination is not uniformly spherical

(a)

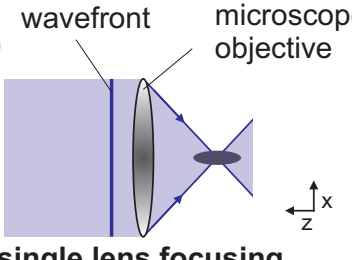

(b)

c)

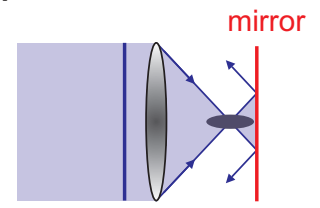

(d)

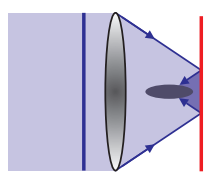

but comes from only one side of the focal point, as illustrated in Fig. 且a. Hence, the best option to date for reducing the axial extension of the spot consists in focusing light in a coherent manner through two objective lenses facing each other, as depicted in Fig. 1 b, so that the illumination is almost spherical [8]. The constructive interference of the two focused light fields produces an intensity peak whose axial dimension is 3 times narrower than that obtained with a single lens. This approach applied to fluorescence microscopy under the name of $4 \mathrm{Pi}$ microscopy, has yielded impressive three-dimensional images with quasi-isotropic resolution [9, 10] but it suffers from serious practical limitations due to the complexity of adjusting the double objective lenses and the double optical paths. In this Letter, we show theoretically and experimentally that isotropic spots similar to that achieved using $4 \mathrm{Pi}$ focusing can be obtained by replacing one of the lenses with a mirror.

Focusing a laser beam before or after a mirror, by itself, cannot bring any improvement of the spot geometry simply because the incident and the reflected fields do not participate together to the spot formation (Figs. 1 $\mathrm{c}$ and 1 d). It is also necessary to shape the incident beam so that both the incident and reflected fields converge toward the same point. Electromagnetic time-reversal cavity theory has recently provided a proper framework for this goal. The theory states that by sending in time reversed order (or with phase conjugation), the field radiated by a point source in an arbitrary environment, one forms an optimal light spot at the source location [6, 11] 13. More precisely, the far-field radiated by a monochromatic electric dipole $\mathbf{p}$ placed at $\mathbf{r}_{0}=\left(0,0, z_{0}\right)$ above a perfect mirror can be decomposed into a sum of plane waves propagating in the $\mathbf{u}$ direction with $\mathbf{u} \cdot \hat{\mathbf{z}}=\cos \theta$ and $|\theta| \leq 90^{\circ}$, see Fig. 2a. The field radiated by one dipole above a mirror is equivalent to the field radiated in free space by two dipoles with opposite transverse components, placed symmetrically with respect to the mirror plane. If $\mathbf{p}$ is parallel to the mirror, one finds that the complex amplitude $\mathbf{e}(\mathbf{u})$ of the plane wave emitted in the
FIG. 1: (Color online). Illumination schemes with (a) a single lens, (b) two lenses facing each other, $(c, d)$ with a single lens and a mirror. Panels (c) and (d) illustrate cases for which the incident and reflected fields do not participate together to the spot formation. 
(a)

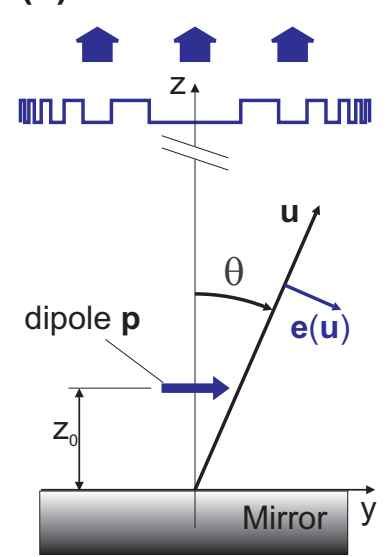

(b)

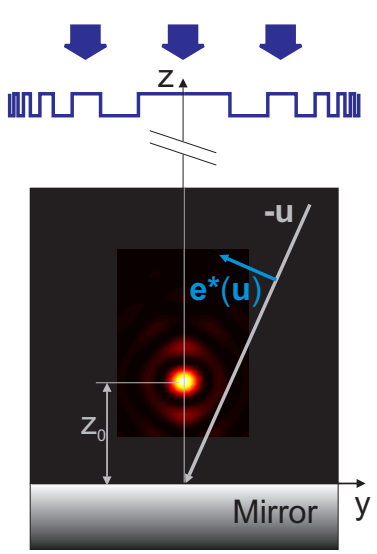

FIG. 2: (Color online). Principles of time-reversal focusing. (a) Radiation of a dipole above a mirror; the blue line depicts the wavefront of the radiated far field. (b) Simulation of the field intensity distribution obtained by illuminating the mirror with a shaped beam whose wave front is the opposite of that of the dipole radiated field.

(a)

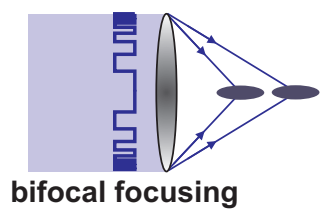

(b)

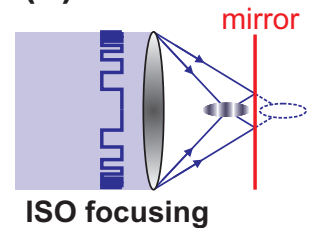

FIG. 3: (Color online). Illumination scheme in the case of ISO focusing. (a) The incident beam is shaped in order to focus simultaneously at two points. (b) By placing a mirror at equidistance between these points, both incident and reflected fields contribute to the spot.

$\mathbf{u}$ direction is given by,

$$
\mathbf{e}(\mathbf{u}) \propto \sin \left[\frac{2 \pi}{\lambda} z_{0} n \cos \theta\right][\mathbf{p}-(\mathbf{p} \cdot \mathbf{u}) \mathbf{u}]
$$

where $\lambda$ is the wavelength of the radiated light in vacuum and $n$ is the refractive index of the medium. 22] The time-reversal theory shows that the field obtained by illuminating the mirror with a beam made of plane waves propagating in all the $-\mathbf{u}$ directions with complex amplitudes $\mathbf{e}^{\star}(\mathbf{u})$ is proportional to the imaginary part of the field emitted by the dipole [Fig. 2b] [13]. Note that sending such a beam in free space would yield two spots separated by $2 z_{0}$ along the optical axis, as illustrated in Fig. 3a. By placing a mirror at equidistance between these spots, one obtains an interference pattern, Fig. $3 \mathrm{~b}$, similar to that achieved with $4 \mathrm{Pi}$ focusing Fig. 1 $\mathrm{b}$. In the following, this new illumination scheme will be referred to as isotropic single objective (ISO) focusing.

We have simulated the field intensity obtained by sending onto a mirror a set $500 \times 180$ plane waves along directions $-\mathbf{u}$, with regular spacing in polar and azimuthal

(a)

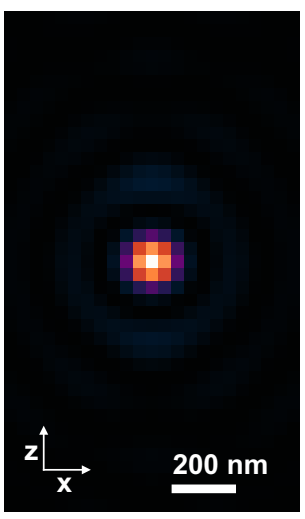

(b)

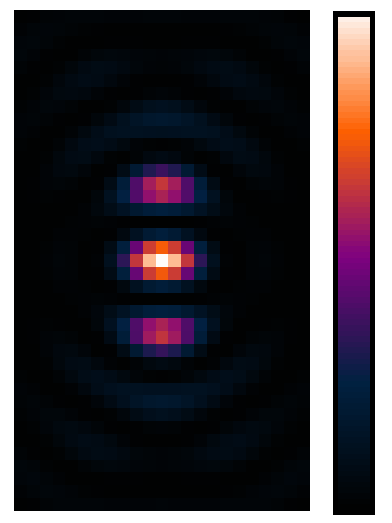

FIG. 4: (Color online). Simulation of the illumination intensity obtained with ISO focusing in water $(n=1.33)$ for $\lambda=491 \mathrm{~nm}$ (a) in the ideal case of $\theta_{\max }=90^{\circ},(\mathrm{b})$ in the case of an objective lens of numerical aperture 1.2, i. e. $\theta_{\max }=64^{\circ}$. In order to be consistent with the experimental conditions, only the phase of the plane waves was shaped.

angles with $\theta \in\left[-\theta_{\max },+\theta_{\max }\right]$ having the phase of $\mathbf{e}^{\star}(\mathbf{u})$ and unit amplitude. We used the vectorial model proposed by Török et al. [14, and assumed the field linearly polarized (along the $y$ axis) in the front focal plane of the objective. For $\theta_{\max }=90^{\circ}$, which corresponds to an ideal microscope objective, one obtains an isotropic spot of diameter about half the wavelength, as depicted in Fig. 4ta. In practice, since $\theta_{\max }<90^{\circ}$, side lobes emerge on both sides of the spot, as illustrated in Fig. $4 \mathrm{~b}$.

To demonstrate experimentally the major interest of the ISO focusing concept, we show, in the following how it can improve the axial resolution of three-dimensional fluorescence microscopy. We have modified a conventional home-built confocal microscope by introducing a phase-only spatial light modulator (SLM) (Pluto-VIS, Holoeye) functioning in reflection between a dichroic mirror (z488/633, Chroma) and a water immersion objective (Plan Apo VC $60 \times$, Numerical aperture = 1.2, Nikon); see Fig. 5. The SLM plane was optically conjugated to the rear focal plane of the objective using a telescope of magnification 2 so that each pixel of the SLM corresponded to one direction $-\mathbf{u}$ in the observation region. The phase pattern was calculated using the time-reversal

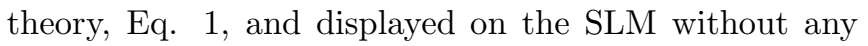
additional correction. Excitation was supplied by a continuous 491-nm laser. The fluorescence light collected in epigeometry was spectrally filtered (FF01-525/39-25, Semrock) and spatially filtered with a $30-\mu \mathrm{m}$ pinhole (i. e., one Airy diameter) placed in front of a photon counter (\$PD1C0C, Micro Photon Devices). Both excitation and fluorescence lights were horizontally polarized (along the $y$ direction). The sample was placed on a nanopositionning stage (NanoLP100, Mad City Labs) which permitted transverse scanning 23. 


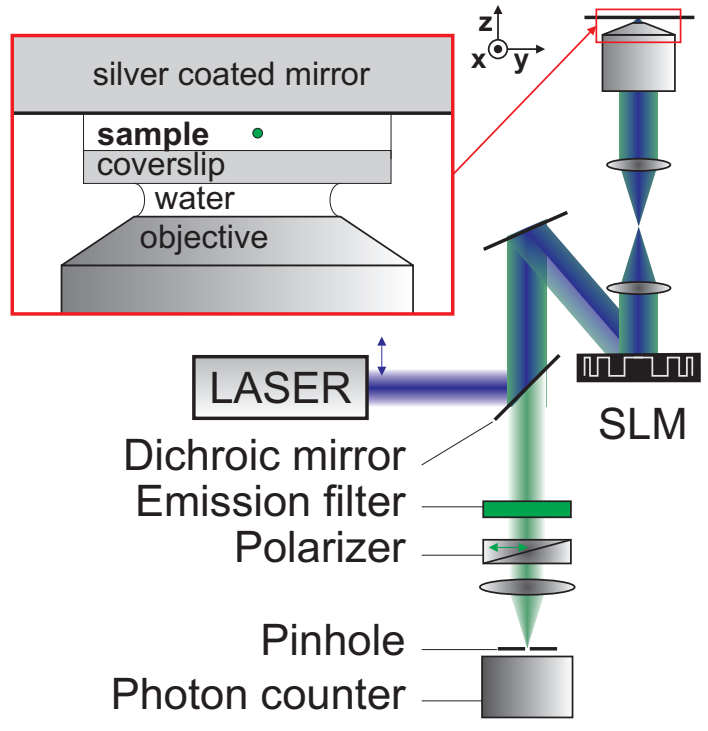

FIG. 5: (Color online). Schematics of the optical setup.

The axial scanning was performed by changing the pattern of the SLM. The axial scanning range was limited to $10 \mu \mathrm{m}$ because the objective was not optimized for focusing far from its focal plane. The conjugation of the pinhole with the focal spot was not affected by the SLM scanning because the fluorescence beam was "descanned" by the SLM. Typical excitation power was $10 \mu \mathrm{W}$. Typical dwell time per pixel was $1 \mathrm{~ms}$. Remarkably, the alignment procedures were not more complicated than those of a conventional confocal microscope and the setup appeared to be quite robust to mechanical drifts or vibration. 24

The axial resolution improvement was assessed by measuring the three-dimensional image of a subwavelength bead. The sample consisted in a suspension of isolated 100-nm spheres (Fluospheres yellow/green, Invitrogen) in a $1 \%$ wt agarose gel and was sandwiched between a Ag coated mirror and a conventional $150-\mu \mathrm{m}$ coverslip.

Slices in the transverse and axial planes of the bead image are displayed in Fig. 6a. As expected, a quasiisotropic central spot surrounded by side lobes is observed. Assuming that the bead is a pointlike object, this image is the product of the incident intensity distribution with the confocal detection point spread function. The axial sectioning capability of the latter permits a reduction of the side lobes amplitudes [9]. A simulation of a pointlike object image obtained with ISO focusing and confocal detection is given in Fig. 6b. We found a good agreement between the experimental data and the simulation. The observed discrepancy is certainly due to a non optimal spot formation, because ISO focusing was performed at a few microns away from the objective focal plane, i. e. where the aberrations are more present. For comparison purposes, acquisitions in conventional confocal mode were also carried out. In this (a)

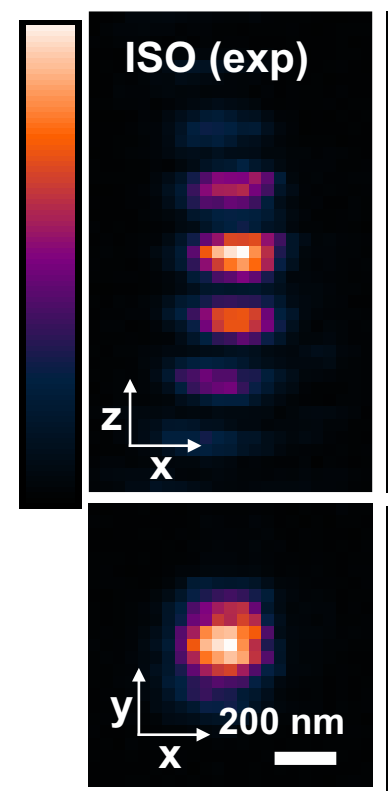

(c)
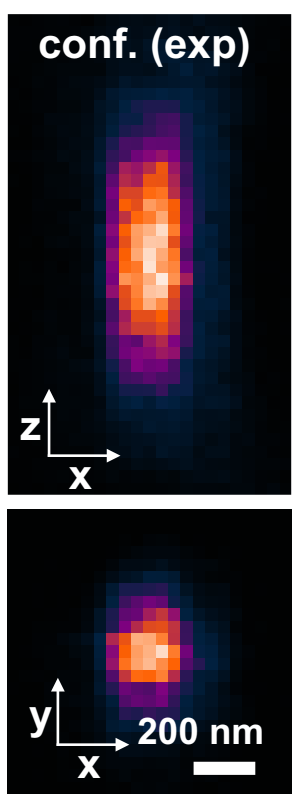

(b)

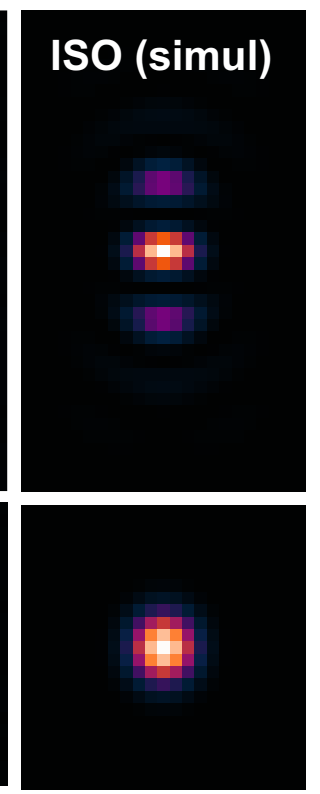

(d)

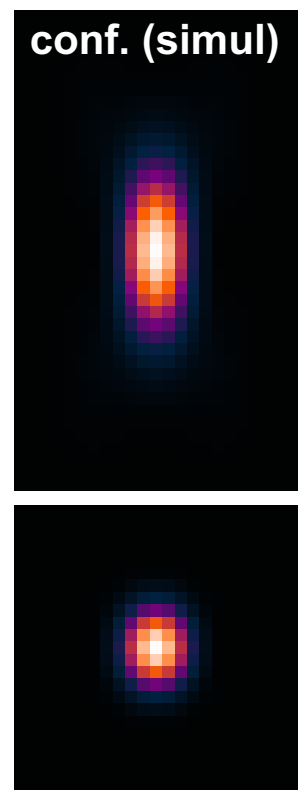

FIG. 6: (Color online). $x z$ and $x y$ slices of the 3D image of an isolated $100 \mathrm{~nm}$ fluorescent bead. (a) ISO focusing, confocal detection, experimental data, (b) ISO focusing, confocal detection, numerical simulation, (c) out of focus confocal mode, experimental data, (d) confocal mode, numerical simulation.

case, the mirror was removed and the SLM was set to generate a single spot at the same defocus as that taken for ISO focusing. The sample was scanned axially with the stage. The image reported in Fig. 6c shows that the experimental spot is slightly larger and longer than that obtained theoretically Fig. 6dd. The issue of focusing 
away from the nominal plane of the objective could be overcome by using specially dedicated objectives or by correcting the aberrations with the SLM.

In conclusion, we have described a simple idea for focusing light into an isotropic diffraction-limited spot using a single objective lens. The ISO focusing concept was applied to confocal microscopy and brought about a dramatic improvement of the axial resolution, similar to that of $4 \mathrm{Pi}$ microscopy, without experimental complexity. All the ameliorations developed for scanning or wide-field $4 \mathrm{Pi}$ microscopy can be readily implemented $[15,16$. ISO focusing opens the way to exciting applications in $3 \mathrm{D}$ imaging as it is compatible with all excitation processes 10, 17 and permits the detection of forward emission 18, 19. Its potential could be further increased by engineering the beam wavefront with more sophisticated techniques [20]. It could then be adapted to a complex focusing environment such as rough or concave substrates, inhomogeneous background 21] or even micro-fluidic devices. More fundamentally, this approach underscores the interest of the time-reversal focusing theory even for very simple configurations.

This work was partially funded by the French Agence Nationale de la Recherche under contract No. ANR-08NANO-P053-36. E. M. and E. L. M. have contributed equally to this work.

* Electronic address: anne.sentenac@fresnel.fr

[1] J. B. Pendry, Phys. Rev. Lett. 85, 3966 (2000).

[2] Z. Liu, H. Lee, Y. Xiong, C. Sun, and X. Zhang, Science 315, 1686 (2007).

[3] A. Sentenac and P. C. Chaumet, Phys. Rev. Lett. 101, 013901 (2008).

[4] F. Lemoult, G. Lerosey, J. de Rosny, and M. Fink, Phys. Rev. Lett. 104, 203901 (2010).

[5] R. Dorn, S. Quabis, and G. Leuchs, Phys. Rev. Lett. 91,
233901 (2003).

[6] S. Quabis, R. Dorn, M. Eberler, O. Glockl, and G. Leuchs, Opt. Commun. 179, 1 (2000).

[7] M. Born and E. Wolf, Principles of Optics (7th (expanded) edn. Cambridge: Cambridge University Press, 1999).

[8] S. Hell and E. Stelzer, J. Opt. Soc. Am. A 9, 2159 (1992).

[9] J. Bewersdorf, R. Schmidt, and S. W. Hell, J. Microsc. (Oxf.) 222, 105 (2006).

[10] R. Schmidt, C. A. Wurm, S. Jakobs, J. Engelhardt, A. Egner, and S. W. Hell, Nat. Methods 5, 539 (2008).

[11] M. Fink, Phys. Today 50, 34 (1997).

[12] G. Lerosey, J. de Rosny, A. Tourin, and M. Fink, Science 315, 1120 (2007).

[13] R. Carminati, R. Pierrat, J. de Rosny, and M. Fink, Opt. Lett. 32, 3107 (2007).

[14] P. Török, P. D. Higdon, and T. Wilson, Opt. Commun. 148, 300 (1998).

[15] M. Gustafsson, D. Agard, and J. Sedat, J. Microsc. (Oxf.) 195, 10 (1999).

[16] S. Hell, R. Schmidt, and A. Egner, Nature Photon. 3, 381 (2009).

[17] M. J. Rust, M. Bates, and X. Zhuang, Nat. Methods 3, 793 (2006).

[18] P. J. Campagnola and L. M. Loew, Nat. Biotechnol. 21, 1356 (2003).

[19] A. Zumbusch, G. R. Holtom, and X. S. Xie, Phys. Rev. Lett. 82, 4142 (1999).

[20] I. M. Vellekoop and A. P. Mosk, Opt. Lett. 32, 2309 (2007).

[21] Z. Yaqoob, D. Psaltis, M. S. Feld, and C. Yang, Nature Photon. 2, 110 (2008).

[22] The horizontal dipole direction was chosen for practical reasons, the Spatial Light Modulator acting on horizontally polarized light.

[23] Transverse scanning by steering the beams with galvanometric mirrors was also tried successfully.

[24] Precise axial positioning of the mirror was achieved by axially scanning the mirror through focus and measuring the detected signal strength. Once the two focal spots were axially localized, the mirror was set at equidistance of the spots with the nanopositionning stage. 